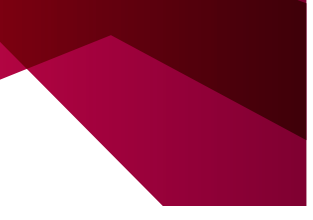

\title{
Recent advances in multiple myeloma: a Korean perspective
}

Junshik Hong and Jae Hoon Lee

Division of Hematology and Medical Oncology, Department of Internal Medicine, Gachon University Gil Medical Center, Incheon, Korea

Received: December 22, 2015 Accepted: August 22, 2016

\section{Correspondence to}

Jae Hoon Lee, M.D.

Department of Internal Medicine, Gachon University Gil Medical Center, 21 Namdong-daero 774beon-gil, Namdong-gu, Incheon 21565, Korea Tel: $+82-32-460-2186$

Fax: $+82-32-460-3233$

E-mail:jhlee@gilhospital.com
Epidemiologically, multiple myeloma (MM) is a malignant disorder of plasma cells with a higher incidence among Western populations than among Asians. However, there is growing evidence of a recent increase in the age-standardized incidence rate (ASR) of MM in Asian countries, particularly Korea. Application of novel agents has resulted in significant improvement of treatment outcomes, and the advances are ongoing with the recent introduction and U.S. Food and Drug Administration's approval of newer agents, including carfilzomib, ixazomib, elotuzumab, and daratumumab. In concert with the technical advances in the cytogenetic and molecular diagnostics of MM, modifications of its diagnosis and staging system have been attempted for better risk stratification. The modified diagnostic criteria from the International Myeloma Working Group in 2014 enabled a strategy of more active treatment for some patients with smoldering MM, with an ultra-high risk of progression, and fine-tuned the definition of end-organ damage, known as CRAB (hypercalcemia, renal insufficiency, anemia, and bone lesions). Considering Korea's trend of aging at an unprecedented rate, we can expect that the ASR of MM will maintain a gradual increase for many years to come; therefore, $\mathrm{MM}$ will be a cancer of critical importance from both medical and socioeconomic perspectives in Korea.

Keywords: Multiple myeloma; Korea; Diagnosis; Neoplasm staging; Epidemiology

\section{INTRODUCTION}

Plasma cell disorders have a spectrum ranging from clinically benign conditions, such as monoclonal gammopathy of unknown significance (MGUS) and smoldering myeloma, to multiple myeloma (MM), an obvious malignancy of plasma cells. Normally, plasma cells are white blood cells that are differentiated from B lymphocytes and secrete large amounts of antibodies.

In patients with $\mathrm{MM}$, uncontrolled proliferation of monoclonal plasma cells induces the overproduction of monoclonal immunoglobulin and causes damage to body organs, including the blood, kidney, and bone, as well as causing substantial immunosuppression.
Since the new millennium, the use of novel agents has contributed to significant improvement in MM treatment $[1,2]$. Advances are ongoing, with the development of next-generation proteasome inhibitors (PIs) and immunomodulatory drugs (IMiDs), as well as the introduction of monoclonal antibodies targeting myeloma cells. Along with the improvement in treatment outcomes, there have been technical advances in the cytogenetic and molecular diagnostics of MM. Therefore, the diagnostic criteria and staging for better risk stratification are continually being modified and revised in the field of MM. In this article, we first briefly review the global and Korean epidemiology of MM, and describe recent advances in the treatment, and changes in the diagnos- 
tics. Finally, we would like to report on the efforts of Korean hematologists thus far, aimed at achieving better clinical outcomes and advances in MM research.

\section{GLOBAL AND KOREAN EPIDEMIOLOGY}

With a world-wide age-standardized incidence rate (ASR) of 1.5/100,000, MM contributes to $1 \%$ of all cancer deaths [3]. MM is most prevalent at ages from 65 to 70 years and is slightly more prevalent in males. The ASR in Western countries is generally higher than that worldwide; in most Western countries, MM is the second most common hematologic malignancy after non-Hodgkin's lymphoma [3,4]. Recently, the U.S. Surveillance, Epidemiology, and End Results (SEER) database reported that the ASR and age-adjusted mortality rates of MM were 6.3/100,000 and 3.3/100,000, respectively [4]. The 5-year survival estimated in the SEER database increased from $25 \%$ in 1975 to $49 \%$ in 2011 , probably as a result of advances in novel anti-myeloma treatment [1] along with improved supportive care.

MM is almost always preceded by MGUS, an asymptomatic pre-malignant stage with a low burden of serum M-protein and plasma cells in the bone marrow [5]. For the diagnosis of MGUS, there should be no symptoms or signs related to organ or tissue damage caused by MM, known as CRAB (hypercalcemia, renal insufficiency, anemia, and bone lesions) features. MGUS is present in roughly $3 \%$ to $4 \%$ of the population aged 50 years and older and increases with age [5-7]. The previously reported risk of progression of MGUS to MM or related plasma cell disorders was approximately $1 \%$ per year [5], and > 1.5 g/dL of serum M-protein, M-protein of the non-immunoglobulin $\mathrm{G}(\mathrm{IgG})$ subtype, and an abnormal free light chain (FLC) ratio are risk factors of the progression to MM [8]. IgM MGUS has a higher risk of progression to plasma cell disorders, mostly Waldenström's macroglobulinemia (1.5\%/year), than the risk of progression to MM from IgG MGUS (1\%/year) or that of light chain MGUS (0.3\%/year) [9]. Park et al. [10] reported that, among 1,118 elderly (age $\geq 65$ years) members of an urban Korean population, the age- and gender-adjusted MGUS prevalence was 3.3\% (95\% confidence interval [CI], 2.0 to 4.6), slightly lower than the Western data. A subsequent Korean study showed that the natural clin- ical course of MGUS, including the rate of progression to $\mathrm{MM}$ in Korea (1\%/year), is similar to that in Western countries [11].

Incidence of MM can differ according to ethnicity. An older study conducted in the United States reported that African Americans had an approximately 2-fold higher ASR $(9.5 / 100,000)$ than Caucasian Americans (4.1/100,000) [12]. According to the latest International Agency for Research on Cancer data, Asian countries have lower ASRs of MM than Western countries [3]. However, there is growing evidence that ASRs are increasing in some Asian regions, including Korea [13], Taiwan [14], and Thailand [15].

According to the 2012 Korean data on national cancer statistics [16], MM comprised 1,272 new cases and 823 deaths in 2012. Its crude incidence rate and crude mortality rate were 2.5/100,000 and 1.6/100,000, respectively, and the ASR and age-standardized mortality rate were 1.6/100,000 and 1.0/100,000, respectively. Until 2012, there was a 2-fold increase in the ASR of MM over the last 10 years, and the increase was striking considering that the ASR of MM in 2012 was 10 times higher than that 20 years ago (Fig. 1) [13]. The incidence of all cancers in Korea has doubled during the last 10 years, while that of acute myeloid leukemia (AML) has stagnated during the same period. As a result, the number of new cases of MM surpassed AML for the first time in 2012 by a narrow margin (1,272 for MM vs. 1,257 for AML) with an identical crude incidence rate (2.5/100,000 for both of them) $[16,17]$. Although the ASR of AML $(2.0 / 100,000)$ was still

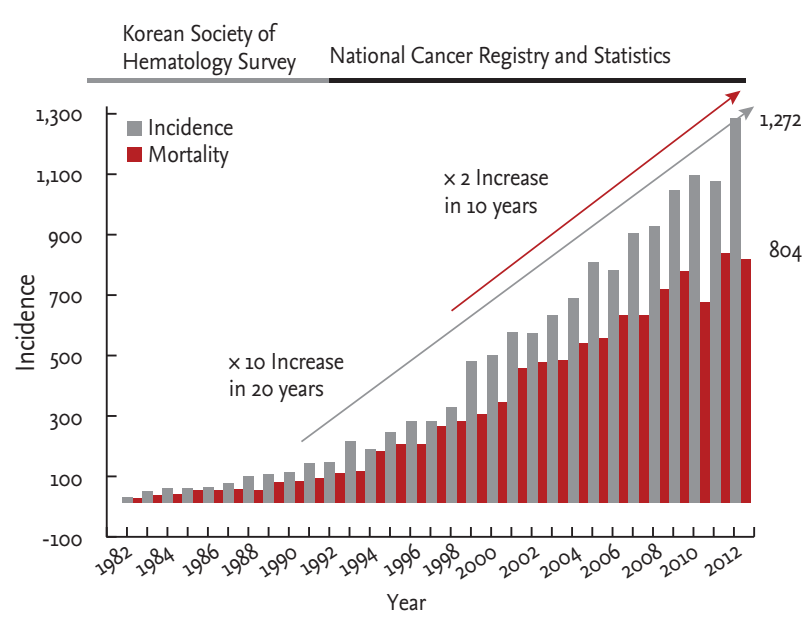

Figure 1. Changes in the age-standardized incidence rate and mortality rate of multiple myeloma in Korea. 
higher than that of MM (1.6/100,000), probably resulting from the skewed distribution of AML toward the elderly population, we can actually regard MM as the second most common hematologic malignancy in Korea considering its recent higher trend of increase compared to AML $[16,17]$. Both improved detection and true increase appear to contribute to the increase in the ASR of MM in Korea. The expansion of health insurance, popularization of medical screening, relevant education of students and surgeons, and public awareness of the disease has enabled more and earlier detection of MM. On the other hand, the genuine increase of MM in Korea can be attributed to exposure to environmental risks, such as air pollution, chemicals, and radiation, all of which are related to rapid industrialization. Furthermore, aging is an important factor affecting this perceived increase of $\mathrm{MM}$, considering that myeloma is most frequently diagnosed among people aged 65 to 74 according to the SEER data. Recently, United States investigators have predicted that MM will primarily be a disease that affects older persons aged 64 to 84 years, and circa 2032 to 2034 , three of every four newly diagnosed MM patients will be aged 64 to 84 years, an increase from up to two of every three patients diagnosed today [18]. According to the life table of Korea, the life expectancy of a Korean baby born in 2014 is 82.4 years [19]. The proportion of the population aged $\geq 65$ years has increased from $7 \%$ in 1999 to $11.8 \%$ in 2012 and is expected to increase to $14 \%$ in 2017 , and to $20.8 \%$ in 2026 [20]. Reflecting Korea's aging, which is proceeding at an unprecedented rate, we can expect that the ASR of MM will maintain a gradual increase for many years to come; therefore, MM will be one of the cancers in the spotlight from both medical and socioeconomic perspectives in Korea.

\section{RECENT IMPROVEMENT IN SURVIVAL}

Systemic therapy with anti-myeloma agents should be applied in patients with newly diagnosed MM, followed by high-dose therapy (HDT) with autologous stem cell transplantation (ASCT) for younger and fit patients. Therefore, the first step in treatment is to determine whether the patient is a candidate for HDT with ASCT, based on age and comorbidities. For patients who are suitable for ASCT, drugs that can compromise stem cell reserve are initially avoided. Consolidative or maintenance therapy has also been applied in post-ASCT patients with MM. By contrast, for patients who are ineligible for ASCT, as well as for patients who have relapsed or progressed after ASCT, effective anti-myeloma regimens are used sequentially, pursuing the prolongation of survival with minimization of treatment-related toxicities.

There are three monumental times in the history of MM treatment. The first meaningful extension of overall survival (OS) was achieved by melphalan in the 1960 s [21]. This 'great old standard agent' is still used in contemporary treatment, in combination with newer agents. The second, HDT with ASCT, has contributed to better treatment outcomes since the 1990s. By increasing the dose intensity, it showed superior OS to conventional chemotherapy $[22,23]$. Although there are some expectations for the omission of ASCT, at least in selected patients who can achieve a durable response to novel agentbased therapy, ASCT has been an obvious standard of care for transplant-eligible patients with MM in the first response [24]. The third evolution of modern MM treatment has been the introduction of novel agents, including PIs and IMiDs. The integration of new agents has resulted in further significant improvement of OS [25].

Thalidomide, an IMiD prohibited in 1961 due to a historical episode of a birth defect crisis, was resurrected as an effective novel agent against MM. In 1999, Singhal et al. [26] conducted a phase II study of thalidomide monotherapy in patients with relapsed or refractory (R/R) MM and reported that thalidomide was active against advanced myeloma with a clinical benefit rate of $32 \%$, and induced marked and durable responses in some patients. Subsequently, it was approved as the frontline induction regimen in combination with dexamethasone (thalidomide plus dexamethasone [TD]) [27]. Bortezomib, the first-generation PI, opened the door to the era of novel agents in earnest. In 2003, based on a pivotal phase II study [28], bortezomib became the second agent of accelerated approval by the U.S. Food and Drug Administration (FDA), next to imatinib mesylate, in the field of hematologic malignancy. In a sequence with more trials as a single agent [29] or combination [30] in the $\mathrm{R} / \mathrm{R}$ setting, it was approved as a first-line therapy for elderly patients with MM in combination with melphalan and prednisone, according to the results of the phase III VISTA study in 2008 [31]. A meta-analysis reported that, 
for transplant-eligible patients, bortezomib-containing induction treatment showed a superior 3-year OS compared to non-bortezomib-based treatment (79.7\% vs. $74.7 \%, p=0.04$ ) [32]. A newer IMiD, lenalidomide, also showed positive results [33,34] and was approved for previously treated patients with MM in 2006. Now, the territory of lenalidomide has been expanded to up-front and maintenance therapy of MM: induction therapy with lenalidomide plus dexamethasone (Rd), followed by lenalidomide maintenance, showed superior median progression-free survival (PFS; 25.5 months vs. 21.1 months, $p=0.001)$ and 4 -year OS (59.4\% vs. $51.4 \%, p=$ 0.0168 ), as compared to therapy with melphalan, prednisone, and thalidomide (MPT) in the phase III FIRST trial [35]. The role of lenalidomide as an effective maintenance agent was reaffirmed among patients who received ASCT [36].

Despite those early successes, the prognosis is poor for patients who are refractory to bortezomib, thalidomide, and lenalidomide [37]. Newer PIs or IMiDs and targeted agents with a novel mechanism of action are strong candidates to overcome those unmet needs. In a phase III MM-oo3 study, pomalidomide, a new third-generation IMiD, showed activity against R/R MM with manageable toxicity: after a median follow-up period of 10.0 months, the median PFS with pomalidomide plus lowdose dexamethasone was 4.0 months versus 1.9 months with high-dose dexamethasone (hazard ratio [HR], o.48; $p<0.0001$ ) [38]. Carfilzomib is a new PI that irreversibly binds to and inhibits the chymotrypsin-like activity of the $20 \mathrm{~S}$ proteasome, which mediates proteolysis [39]. The phase III ASPIRE trial compared carfilzomib, lenalidomide, and dexamethasone to Rd. A remarkable 9-month improvement in the median PFS was achieved with carfilzomib (26.3 months vs. 17.6 months; HR, 0.69; $p=0.0001$ ), and improvement was shown in the response rate, depth of response, and patient-reported quality of life (QoL) metrics, with tolerable toxicity [40]. In the randomized open-label phase III ENDEAVOR study, which enrolled 979 patients globally, carfilzomib with dexamethasone nearly doubled the median PFS compared with bortezomib with dexamethasone for R/R MM (18.7 months vs. 9.4 months; HR, 0.53; $p<0.0001$ ) [41]. In November 2015, the FDA approved ixazomib, a once-weekly oral PI, indicated in the combination with $\mathrm{Rd}$ for the treatment of MM patients who have had at least one pri- or therapy, based on the phase III TOURMALINE-MM1 trial [42]. Those two newer PIs have advantageous toxicity profiles characterized by a low rate of peripheral neuropathy compared with bortezomib. In particular, fully oral PI-based combination therapy would improve the patients' convenience by the integration of ixazomib [43]. According to the result of a global multicenter phase III study, panobinostat, a pan-deacetylase inhibitor and epigenetic modulator targeting both class I and II histone deacetylase enzymes, could be a useful addition to the treatment with bortezomib and dexamethasone for patients with R/R MM [44]. Elotuzumab is an immunostimulatory monoclonal antibody that targets signaling lymphocytic activation molecule F7. In a phase III clinical trial, in combination with the Rd regimen, elotuzumab reduced the risk of disease progression or death by $30 \%$ in patients with R/R MM [45], leading to FDA approval in November 2015. Daratumumab is a CD38-targeting, human IgGik monoclonal antibody. Reflecting a promising outcome, a 36\% response rate as a single agent in a phase I-II study involving patients with R/R MM [46], the FDA granted accelerated approval in November 2015. By advances from R/R patients to an up-front setting, those newer PIs, IMiDs, and targeted agents would lead to further improvement in the OS of $\mathrm{MM}$, as well as better QoL because of improved toxicity profiles compared with earlier novel agents.

\section{RECENT MODIFICATION OF THE DIAGNOSTIC CRITERIA}

In 2014, The International Myeloma Working Group (IMWG) recommended updated criteria for the diagnosis of MM [9]. Unlike other hematologic malignancies, the disease definition of MM is characteristically clinicopathological and overt clinical manifestations of end-organ damage attributable to MM, known as CRAB features, have been used in classifying patients as smoldering MM and MM requiring therapy. However, modification is required due to the advances in anti-myeloma therapy with novel agents; with the newer agents, early intervention in high-risk asymptomatic patients can extend survival [47]. Furthermore, as laboratory and radiological techniques have progressed in the last decade, there is growing evidence that some biomarkers should 
be regarded as CRAB features that define MM requiring therapy [48]. Therefore, compared with previous IMWG criteria reported in 2003 [49], the updated recommendation included validated biomarkers in addition to the existing requirements of attributable CRAB features. In addition, a small portion of patients previously classified as smoldering MM but with biological malignancy and imminent risk of progression, the so-called 'ultra-high risk group' in smoldering MM, were classified as MM.

Smoldering MM, which denotes patients of MM who demonstrate the proliferation of malignant plasma cells and a subsequent overabundance of monoclonal paraprotein, but who are devoid of myeloma-related organ or tissue impairment, is a very slow-growing type of myeloma [50]. According to an analysis of 276 patients with smoldering MM for a 26-year period at the Mayo Clinic, approximately one half of patients with smoldering MM were free of progression during the first 5 years, and roughly $30 \%$ did not progress even after 10 years [51]. However, not all patients with smoldering MM show an indolent course. Studies defined a small cohort of patients with smoldering $\mathrm{MM}$ who have a notably high risk of early progression [52]. Reflecting this fact, IMWG reached a consensus that, if reliable biomarkers associated with approximately $80 \%$ probability of progression to MM within 2 years were identified, such patients should be regarded as having MM and treatment should be offered [9]. The meaning and intent of this re-classification is that, for patients with ultra-high risk smoldering $\mathrm{MM}$, delaying treatment until fulfilling the criteria of symptomatic MM would result in end-organ damage causing the impairment of QoL as well as inferior OS that could otherwise be avoidable with the application of adequate early intervention.

There are several suggested myeloma-defining biomarkers in the 2014 IMWG modification. First, bone marrow plasma cells (BMPCs) $\geq 60 \%$ was used as a marker of MM requiring therapy. This modification is based on the result of a Mayo Clinic study showing that 21 of 655 analyzed patients with smoldering MM with BMPCs $\geq 60 \%$ had a $95 \%$ risk of progression within 2 years of diagnosis (median time to disease progression, 7.0 months; 95\% CI, 1.0 to 12.9) [53], and validated in another study [54]. The second biomarker indicative of symptomatic MM is an involved: uninvolved serum-free light-chain ratio $\geq 100$. A retrospective study analyzing
586 patients with smoldering MM reported a ratio of at least 100 in 90 patients (15\%), and their risk of progression to $\mathrm{MM}$ or AL amyloidosis within 2 years was 79\% [55]. This finding was reproduced by a study conducted by Kastritis et al. [54]. Finally, patients previously defined with smoldering MM with > 1 focal lesion on magnetic resonance imaging (MRI) studies will be considered MM. Hillengass et al. [56] evaluated 149 patients with smoldering MM with whole-body MRI. Focal lesions were detected in 42 patients (28\%), and 23 patients (15\%) had > 1 focal lesion. The presence of $>1$ focal lesions showed an association with an increased risk of progression in univariate analysis ( $\mathrm{HR}, 4.05 ; \mathrm{p}<0.001$ ) and maintained predictive value in multivariate analysis. For those patients, the median time to progression (TTP) was 13 months, and 70\% experienced disease progression at 2 years [56]. In this MRI-based 2014 modified diagnostic criterion of MM, each focal lesion must be $5 \mathrm{~mm}$ or more in size. Smaller focal lesions should be evaluated with additional imaging such as computed tomography (CT) or positron emission tomography (PET)/ CT scan. Although reports have suggested that diffuse marrow infiltration in MRI among patients with smoldering $M M$ is predictive of progression to $M M$, it is not recommended as a classification-modifying biomarker, and additional data are required $[56,57]$.

The definitions of end-organ damage (CRAB) were also modified. First, regarding bone disease, it is regarded as CRAB if a patient has osteolytic lesions or osteoporosis with compression fractures attributable to a plasma cell disorder. Conventional skeletal radiography has been used for the evaluation of bone disease. In a systematic review of 32 studies comparing modern imaging modalities, including MRI, PET/CT, and whole-body CT versus whole-body skeletal radiography, by Regelink et al. [58], newer imaging techniques showed greater sensitivity for the detection of bone lesions, with $\geq 80 \%$ of lesions detected by MRI, PET/CT, or whole-body CT. Therefore, the updated IMWG criteria clarified that $\geq$ 2 sites of osteolytic bone lesions ( $\geq 5 \mathrm{~mm}$ in size) on CT or PET/CT scan fulfill the CRAB requirements irrespective of the skeletal radiography findings. In addition, the presence of osteoporosis or vertebral compression fractures without lytic bone lesions is no longer regarded as CRAB, avoiding over-diagnosis among elderly patients in whom osteoporosis and compression fracture 
are common. Second, the definition of renal failure was revised. The concentration of serum creatinine differs widely based on age, gender, and race. Therefore, the modified criteria recommended that the measured or estimated glomerular filtration rates, according to either the modification of diet in renal disease or the chronic kidney disease epidemiology collaboration formula, less than $40 \mathrm{~mL} / \mathrm{min}$ should be used in addition to a fixed $173 \mu \mathrm{mol} / \mathrm{L}(>2 \mathrm{mg} / \mathrm{dL}$ ) serum creatinine. The IMWG also stated that only renal insufficiency caused by lightchain cast nephropathy is considered a myeloma-defining event and recommended a kidney biopsy to clarify the underlying cause of renal insufficiency in patients with suspected cast nephropathy, particularly if the serum-involved FLC levels are less than $500 \mathrm{mg} / \mathrm{L}$, in line with the recommendations of the International Kidney and Monoclonal Gammopathy Research Group [59].

In the modified definition of MM, the requirement for the presence of monoclonal protein is not mandatory because some patients with MM have no detectable abnormalities on serum or urine immunofixation (so called 'non-secretory' MM), but they fulfill other required criteria of MM and show no difference in the biological and clinical characteristics compared with patients with secretory MM [49]. In addition, the minimal requirement of BMPC was clearly stated as $10 \%$, to prevent patients with MGUS from being wrongly classified as MM based on the features of anemia, renal insufficiency, or hypercalcemia that are, in such a case, not attributable to MM. A schematic summary of key points in the diagnosis of plasma cell disorders, including MM, according to the
2014 IMWG modification is shown in Table 1.

\section{EFFORTS FOR BETTER RISK STRATIFICATION}

Since the replacement of the Durie-Salmon staging system, the International Staging System (ISS) has been used by virtue of its simplicity and better prognostication. A weakness of the ISS is that it does not consider cytogenetics. For this reason, efforts have been made to establish a better staging system. A retrospective study using the IMWG database of 12,137 patients with MM treated worldwide evaluated the potential role of combining ISS stage and cytogenetics for the prediction of survival. Among 2,642 patients with sufficient interphase fluorescence in situ hybridization (iFISH) data, the combination of $\mathrm{t}(4 ; 14)$ or deletion (17p) to ISS stage and age significantly improved the prognostic power in terms of the PFS and OS [6o]. Palumbo et al. [61] combined the ISS with chromosomal abnormalities detected by iFISH after $\mathrm{CD} 138$ plasma cell purification and serum lactate dehydrogenase $(\mathrm{LDH})$ for the evaluation of their prognostic value in newly diagnosed MM. Their revised ISS (R-ISS) defined R-ISS stage I as patients with ISS stage I plus no high-risk chromosomal abnormality [del(17p) and/or t(4;14) and/or t(14;16)] and a normal serum LDH level. R-ISS stage III was defined as patients with ISS stage III plus either high-risk chromosomal abnormality by iFISH or elevated LDH. All of the other patients were classified as R-ISS stage II. At a median follow-up period of 46 months, the 5 -year OS rate was $82 \%$ in the

Table 1. Diagnosis of MGUS, smoldering myeloma, and multiple myeloma according to the International Myeloma Working Group updated criteria for the diagnosis of multiple myeloma in 2014

\begin{tabular}{|c|c|c|}
\hline MGUS & Smoldering myeloma & Multiple myeloma \\
\hline M protein $<3 \mathrm{~g} / \mathrm{dL}$ & M protein $\geq 3 \mathrm{~g} / \mathrm{dL}$ (serum) & $\geq 10 \%$ clonal plasma cells or $\geq 1$ biopsy proven plasmacytoma \\
\hline Clonal plasma cells in & or $\geq 500 \mathrm{mg} / 24$ hours (urine) & And one or more the following myeloma defining events \\
\hline bone marrow $<10 \%$ & Clonal plasma cells in bone marrow & $\geq 1 \mathrm{CRAB}^{\mathrm{a}}$ feature \\
\hline \multirow{3}{*}{$\begin{array}{l}\text { No myeloma defining } \\
\text { events }\end{array}$} & $\geq 10$ but $<60 \%$ & $>_{1}$ focal lesion on magnetic resonance imaging \\
\hline & No other myeloma defining events & Involved: uninvolved serum free light chain ratio $\geq 100$ \\
\hline & & Clonal plasma cells in bone marrow $\geq 60 \%$ \\
\hline
\end{tabular}

MGUS, monoclonal gammopathy of unknown significance; CRAB, calcium, renal insufficiency, anemia, and bone lesion. ${ }^{a}$ Hypercalcemia as serum calcium $>1 \mathrm{mg} / \mathrm{dL}$ higher than the upper limit of normal or $>11 \mathrm{mg} / \mathrm{dL}$, serum creatinine $>2 \mathrm{mg} / \mathrm{dL}$ or creatinine clearance $<40 \mathrm{~mL} /$ minute (newly included in the updated criteria), hemoglobin value of $>2 \mathrm{~g} / \mathrm{dL}$ below the lower limit of normal or a $<10.0 \mathrm{~g} / \mathrm{dL}$, and one or more osteolytic lesions on computed tomography or positron emission tomography/computed tomography (newly included in the updated criteria), as well as skeletal radiography. 
R-ISS I, $62 \%$ in the R-ISS II, and $40 \%$ in the R-ISS III groups. They concluded that this simple and powerful R-ISS could be used in future clinical studies to stratify patients with newly diagnosed MM [61]. Trials to establish more accurate risk stratification are ongoing. For example, a Korean retrospective study showed the prognostic impact of inflammatory factors in patients with MM treated with thalidomide-containing therapy [62]. A cytogenetics-based risk adapted treatment was proposed by investigators at the Mayo Clinic (Mayo Stratification for Myeloma And Risk-adapted Theraopoy 2.0; mSMART 2.0) [63]. The IMWG proposed a risk stratification system reflecting current ISS, FISH, and age and the recommendation for clinical practice (Table 2) [64]. Recently, the incorporation of gene expression profiling showed superiority to current markers by offering a robust prognostic modeling [65].

Besides the assessment of risk according to the biological characteristics of MM, clinical evaluation using geriatric assessment (GA) predicts the survival and risk of toxicity in patients who are not eligible for HDT with ASCT. In a recent pooled analysis of 869 elderly patients with newly diagnosed MM [66], baseline GA was performed, and the patients were classified according to three groups: fit, intermediate, and frail. The 3-year OS was $84 \%$ in fit, $76 \%$ in intermediate (HR, $1.61 ; p=0.042)$, and $57 \%$ in frail (HR, 3.57; $p<0.001)$ patients. GA also predicted the cumulative incidence of grade 3 or higher non-hematologic toxicities and the discontinuation of treatment at 12 months [66]. Based on the results, the IMWG proposed the measurement of frailty in designing future clinical trials for elderly patients.

\section{KOREAN STUDIES ON MULTIPLE MYELOMA}

In Korea, since the first case report of MM in 1959 [67], only a few case reports were published, sporadically, until the 1970s. In the era of conventional chemotherapy, Korean hematologists conducted small-scale studies on $\mathrm{MM}$, and the results were published in domestic journals in the Korean language. Because MM was a relatively rare disease and socioeconomic conditions, including the National Health Insurance had not matured during that period, the studies only reported the clinical features of patients [68-70], or were repeats of Western studies testing the efficacy and tolerability of the already known regimens of conventional cytotoxic agents [71-73]. The treatment outcomes were similar or slightly inferior to those reported by Western studies, probably due to the later time of diagnosis in their disease courses.

Entering the era of HDT with ASCT, Korean hematologists were motivated to conduct multicenter trials of new drugs or to invent creative, new therapeutic strategies to overcome unsatisfactory outcomes and, as a result, they leapt into world class standards of care and research on MM. Spontaneously, small study groups emerged based on geographic distribution or personal acquaintance for the study of MM. They began to conduct small multicenter studies, initially as a retrospective analysis testing the feasibility of ASCT [74], followed by a prospective trial [75]. A more sophisticated prospective trial of risk-adapted therapy based on FISH and serum $\beta 2$-microglobulin results, in which allogeneic transplantation is included for the high-risk group, was attempted [76]. Later, the efficacies of bortezomib [77] or

Table 2. Proposed risk stratification of multiple myeloma and recommended guidelines from the International Myeloma Working Group

\begin{tabular}{|c|c|c|c|}
\hline & Parameter & Median OS, yr & \% Patients \\
\hline High risk & ISS II/III and $\mathrm{t}(4 ; 14)$ or 17p13 del & 2 & 20 \\
\hline Standard risk & Others & 7 & 60 \\
\hline Low risk & ISS I/II and absence of $\mathrm{t}(4 ; 14), 17 \mathrm{p} 13$ del and $+1 \mathrm{q} 21$ and age $<55$ years & $>10$ & 20 \\
\hline
\end{tabular}

Guidelines for clinical practice. (1) The combination of ISS and t(4;14), 17p13, and 1q21 by fluorescent in situ hybridization should be used for risk stratification. The median OS of patients, with high-risk patients surviving about 2 years, despite current novel agent-containing treatment, whereas low-risk patients can survive $>10$ years. (2) This risk stratification system should be used in clinical practice and as the standard for comparison in future studies for prognostic biomarkers, and in clinical trials. (3) Currently, there is no evidence for a risk-adapted treatment approach with the exception that prolonged proteasome inhibitor-based treatment should be given to patients with $\mathrm{t}(4 ; 14)$, and possibly 17p13 deletion.

OS, overall survival; ISS, International Staging System. 
thalidomide [78] in patients with R/R MM were evaluated retrospectively in small numbers of patients, with the advent of novel agents in Korea. Multicenter studies for translational research on MM also began [79].

Despite such progress, there was no nationwide study group. In 2005, the Korean Society of Hematology (KSH) decided to establish official working parties for major hematologic disorders, and the Korean Multiple Myeloma Working Party (KMMWP) was established as one of the 11 working parties of KSH. The first work of KMMWP was a retrospective study evaluating the toxicities of bortezomib in Korean patients with MM. It was the first study to examine this topic among an Asian population [8o]. Overall, Koreans had similar toxicity profiles of bortezomib to those of Western populations, with a common incidence of $47 \%$ thrombocytopenia and $42 \%$ sensory neuropathy. Interestingly, the incidence of gastrointestinal toxicity was lower than in the Western data.

Thereafter, various treatment regimens for MM have been evaluated by Korean investigators. For the frontline induction therapy of transplant-eligible patients, two cycles of bortezomib, thalidomide and dexamethasone (VTD) followed by two cycles of the vincristine, doxorubicin and dexamethasone (VAD) regimen resulted in a response rate of $96 \%$, including a $\geq 28 \%$ rate near complete response (CR) before ASCT [81]. In another phase II study conducted by KMMWP, four cycles of cyclophosphamide with thalidomide and dexamethasone (CTD) showed a response rate of $86.5 \%$, including a CR of $38 \%$ [82]. We then designed a phase II study of sequential high-dose dexamethasone, then response adapted bortezomib, adriamycin, and dexamethasone (PAD) versus $\mathrm{VAD}$ induction chemotherapy followed by $\mathrm{HDT}$ with ASCT for newly diagnosed MM [83]. In the study, we reported that approximately half of the patients who responded to high-dose dexamethasone could be saved with novel agents during induction treatment, and PAD can successfully rescue the other half, who were not sensitive to dexamethasone.

For transplant-ineligible patients, we designed and performed six cycles of VTD induction therapy followed by eight cycles of MPT consolidation therapy. It yielded a $95 \%$ response rate, including a CR rate of $74 \%$ [84]. Using our nationwide registry, we reported that an early response to bortezomib plus chemotherapy can help to predict survival in patients with MM who are not ASCT candidates [85].

For salvage treatment, six cycles of PAD followed by 12 cycles of consolidative thalidomide with dexamethasone resulted in a $\geq 70.2 \%$ very good partial response (VGPR), and the median PFS was 18 months ( $95 \%$ CI, 9.7 to 26.2) and median OS was 35.1 months ( $95 \%$ CI, 18.5 to 51.7 ) during the median follow-up period of 27 months [86]. KMMWP evaluated the efficacy and safety of a four-drug combination of bortezomib plus CTD in a R/R setting: the study reported an overall response rate of $87.2 \%$, including a $45.7 \%$ CR and $8.6 \%$ VGPR, and the 3-year PFS and OS were $14.5 \%$ and $47.2 \%$, respectively [87]. In 2014, we reported our experience of Rd treatment in 110 heavily pretreated R/R MM patients [88]. The overall response rate was $43.6 \%$ with $\geq 15.4 \%$ VGPR, and the median TTP and OS were 8.0 and 23 months, respectively. The toxicities were comparable to the data published in Western countries.

Clinical studies to improve the outcomes of hematopoietic stem cell transplantation have been conducted by KMMWP. According to our retrospective study, achieving at least near CR before ASCT is of critical importance for better results [89]. Patients who attained $\geq$ near CR before ASCT showed a significantly superior survival rate to those who achieved $\geq$ near CR only after ASCT $(p=0.018)$. We compared the results of reduced intensity allogeneic stem cell transplantation (RIST) following ASCT versus tandem ASCT [90]. No significant differences in the event-free survival $(p=0.26)$, OS $(p=0.13)$, treatment-related mortality $(p=0.35)$, and disease-related mortality $(p=0.33)$ were observed between ASCT followed by the RIST group $(\mathrm{n}=30)$ and the tandem ASCT group $(n=126)$.

Gradually, the numbers of participating institutions have increased, and the quality of studies has also improved. The protocols of KMMWP were registered at the National Institute of Health of USA (http://ClinTrials.gov) and were posted to the website of the Multiple Myeloma Research Foundation. The clinical trials of KMMWP were actually the first studies under strict monitoring for quality control in the history of Korean hematology study. The majority of earlier works by KMMWP were the first studies reporting the efficacy of novel agents in the Asian area, and our trials used FISH and conventional cytogenetics analyzed in the central laboratory. In addition, we constructed the Korean Myelo- 
ma Registry (KMR), a web-based patient registry system for future cohort-based studies. Currently, more than 6,60o patients with MM and related plasma cell diseases since the year 2000 have been registered, from over 40 institutions in the KMR. Using the KMR, we have documented the characteristics of this population [91], and subsequent registry-based studies have been published $[85,92]$.

Studies of KMMWP were not limited to clinical trials for anti-myeloma treatment. We evaluated certain clinical or biologic characteristics of clinical significance among patients with MM, including malignant pleural effusion [93], IgD MM [94], Waldenström's macroglobulinemia [95], and primary systemic light-chain amyloidosis [96]. Treatment-related complications among Korean patients, including osteonecrosis of the jaw [97], herpes zoster infection $[98,99]$, and MM-associated venous thrombotic or embolic events (VTE) [100], were also investigated. The VTE study reported a lower incidence (3.9\% of 360 patients) compared with that in Western reports, suggesting an ethnic difference in the susceptibility to VTE.

Only a few basic or translational research studies were conducted by KMMWP. We published the cytogenetic characteristics of Korean patients with MM using FISH and found that 139 deletion (45.6\%) followed by $\operatorname{IgH}$ translocation (41\%) and $1 \mathrm{q}$ gain (38.8\%) were the most common cytogenetic aberrations in Korean patients [101]. Multicenter basic studies in Korea reported the incidence rate of genetic polymorphisms of NQO1 [102] and CYPA1 gene [103], possibly suggesting a lower genetic susceptibility for MM in Asians than in Western populations. We also reported the antagonistic interaction of polyphenols in bortezomib treatment [104], and the incidence of interleukin- 6 receptor gene amplification and its association with prognosis [105]. In our methylation-specific polymerase chain reaction study using two different primer sets, a concurrent p16 methylation pattern was an adverse prognostic factor in patients with MM [106]. Antigen-presenting cells such as dendritic cells (DCs) or CD40-activated B cells were investigated by the members of KMMWP [107,108]. They reported the possibility of immunotherapy for MM using myeloma-specific cytotoxic $T$ cells stimulated in vitro by DCs pulsed with purified and optimized myeloma lysates [107]. The significant defective function of DCs was caused by loading tumor antigens, and the neutralization of vascular endothelial growth factor could overcome this DC dysfunction through the elimination of abnormal signal transduction [109]. Furthermore, autologous DCs loaded with allogeneic myeloma cells have the capacity to produce potent myeloma-specific CTL responses against autologous myeloma cells [110]. We recently reported that chromosome 13 deletion and hypodiploidy on conventional cytogenetics are robust prognostic factors in Korean patients with MM [92].

We also participated in global projects sponsored by the International Myeloma Foundation, including a historical control project [37], a FISH study incorporated with ISS [111] and a recent long-term CR project (ongoing study), as well as some review articles [112,113]. Each of the associated institutions in Korea are actively participating in globally important sponsor-initiated global clinical trials of novel agents beyond the targeted agents of the early period, and substantial numbers of investigator-initiated trials are being conducted in Korea. In some of these studies, Korea contributed significantly to the enrollment, including the PANORAMA-1 trial [44], and Korean specialists also acted as principal investigators, such as in the CLARION trials, which compared carfilzomib with melphalan and prednisone to bortezomib with melphalan and prednisone.

In 2011, we launched the Asian Myeloma Network (AMN) with six neighbor countries and regions. Our first task was to characterize Asian myeloma to determine whether there are unique characteristics. In an analysis of 3,405 patients, collected evenly from seven countries, it was concluded that there are no unique Asian-specific findings regarding the demographics, clinical findings, cytogenetic characteristics, and prognostic indices [114]. In addition, we participated in Asian guidelines for the management of MM, which is a resource-stratified guideline [115]. This is regarded as an important guideline, even outside Asia, where developing countries are facing limited resources in diagnostic and therapeutic facilities, especially novel agents. We sought collaboration in retrospective and prospective studies among AMN countries [116] as well as with United Stats institutes [117]. In these studies, we compared the upfront use of novel agents with salvage use between a Korean database and a Singapore database, and evaluated lenalidomide monotherapy for newly diagnosed 
standard-risk MM at Korean sites and the Lee Moffitt Cancer Center of the United States.

We also initiated some investigator-initiated trials using novel agents, including pomalidomide and carfilzomib, inside AMN. We are now seeking active collaboration with numerous study groups, including the European Hematology Association, Greek Myeloma Study Group, Australian Myeloma and Related Disease Registry, as well as the Latin America Myeloma Group. Hopefully, the research activities on MM in Korea and Asian countries that have been active in recent years will progress to the world stage, resulting in collaboration with the United States and European Groups.

\section{CONCLUSIONS}

Recent advances in the treatment of MM, after the introduction of novel agents, have resulted in an improved survival rate that has been more than twice that of the past. This improvement, in such a short period, is an unprecedented achievement in the history of hematologic malignancy. It is believed that we are now curing at least a fraction of MM patients. The prognosis of MM will improve further with the advent of newer classes of novel drugs, such as monoclonal antibodies and adoptive cellular therapies. Korea is a good model for MM research, having demonstrated how it can evolve through collaborative effort among investigators at the domestic and international levels. By virtue of these efforts, we anticipate that MM will become a curable disease in the near future, given the understanding of novel prognostic factors as well as the introduction of newer, targeting agents.

\section{Conflict of interest}

No potential conflict of interest relevant to this article was reported.

\section{REFERENCES}

1. Kumar SK, Rajkumar SV, Dispenzieri A, et al. Improved survival in multiple myeloma and the impact of novel therapies. Blood 2008;111:2516-2520.

2. Kumar SK, Dispenzieri A, Lacy MQ, et al. Continued im- provement in survival in multiple myeloma: changes in early mortality and outcomes in older patients. Leukemia 2014;28:1122-1128.

3. Ferlay J, Soerjomataram I, Dikshit R, et al. Cancer incidence and mortality worldwide: sources, methods and major patterns in GLOBOCAN 2012. Int J Cancer 2015;136:Е359-E386.

4. Siegel RL, Miller KD, Jemal A. Cancer statistics, 2015. CA Cancer J Clin 2015;65:5-29.

5. Kyle RA, Therneau TM, Rajkumar SV, et al. A long-term study of prognosis in monoclonal gammopathy of undetermined significance. N Engl J Med 2002;346:564-569.

6. Kyle RA, Therneau TM, Rajkumar SV, et al. Prevalence of monoclonal gammopathy of undetermined significance. N Engl J Med 2006;354:1362-1369.

7. Blade J. Clinical practice: monoclonal gammopathy of undetermined significance. N Engl J Med 2006;355:27652770.

8. Kyle RA, Durie BG, Rajkumar SV, et al. Monoclonal gammopathy of undetermined significance (MGUS) and smoldering (asymptomatic) multiple myeloma: IMWG consensus perspectives risk factors for progression and guidelines for monitoring and management. Leukemia 2010;24:1121-1127.

9. Rajkumar SV, Dimopoulos MA, Palumbo A, et al. International Myeloma Working Group updated criteria for the diagnosis of multiple myeloma. Lancet Oncol 2014;15:e538-e548.

10. Park HK, Lee KR, Kim YJ, et al. Prevalence of monoclonal gammopathy of undetermined significance in an elderly urban Korean population. Am J Hematol 2011;86:752-755.

11. Lee YG, Bang SM, Lee JO, et al. Five-year follow-up study of monoclonal gammopathy of undetermined significance in a Korean elderly urban cohort. Cancer Res Treat 2015;47:215-220.

12. Riedel DA, Pottern LM. The epidemiology of multiple myeloma. Hematol Oncol Clin North Am 1992;6:225-247.

13. Lee JH, Lee DS, Lee JJ, et al. Multiple myeloma in Korea: past, present, and future perspectives. Experience of the Korean Multiple Myeloma Working Party. Int J Hematol 2010;92:52-57.

14. Huang SY, Yao M, Tang JL, et al. Epidemiology of multiple myeloma in Taiwan: increasing incidence for the past 25 years and higher prevalence of extramedullary myeloma in patients younger than 55 years. Cancer 2007;110:896905 . 
15. Khuhaprema T, Attasara P, Sriplung H, Wiangnon S, Sangrajrang S. Cancer in Thailand. Vol. 7. Bangkok: Ministry of Public Health and Ministry of Education, Thailand, 2013.

16. Jung KW, Won YJ, Kong HJ, et al. Cancer statistics in Korea: incidence, mortality, survival, and prevalence in 2012. Cancer Res Treat 2015;47:127-141.

17. Park EH, Lee H, Won YJ, et al. Nationwide statistical analysis of myeloid malignancies in Korea: incidence and survival rate from 1999 to 2012. Blood Res 2015;50:204-217.

18. Rosenberg PS, Barker KA, Anderson WF. Future distribution of multiple myeloma in the United States by sex, age, and race/ethnicity. Blood 2015;125:410-412.

19. Statistics Korea. Life table 2014 [Internet]. Daejeon (KR): Statistics Korea, c1996 [cited 2016 Aug 22]. Available from: http://kostat.go.kr/portal/korea/kor_nw/2/2/7/index. board?bmode=read\&aSeq=350178.

20. Hyun KR, Kang S, Lee S. Population aging and healthcare expenditure in Korea. Health Econ 2015 Jun 16 [Epub]. http://dx.doi.org/10.1002/hec.3209.

21. Hernandez JM, Garcia-Sanz R, Golvano E, et al. Randomized comparison of dexamethasone combined with melphalan versus melphalan with prednisone in the treatment of elderly patients with multiple myeloma. Br J Haematol 2004;127:159-164.

22. Attal M, Harousseau JL, Stoppa AM, et al. A prospective, randomized trial of autologous bone marrow transplantation and chemotherapy in multiple myeloma. Intergroupe Francais du Myelome. N Engl J Med 1996;335:9197.

23. Child JA, Morgan GJ, Davies FE, et al. High-dose chemotherapy with hematopoietic stem-cell rescue for multiple myeloma. N Engl J Med 2003;348:1875-1883.

24. Moreau P, Attal M. All transplantation-eligible patients with myeloma should receive ASCT in first response. Hematology Am Soc Hematol Educ Program 2014;2014:250254.

25. Orlowski RZ. Novel agents for multiple myeloma to overcome resistance in phase III clinical trials. Semin Oncol 2013;40:634-651.

26. Singhal S, Mehta J, Desikan R, et al. Antitumor activity of thalidomide in refractory multiple myeloma. N Engl J Med 1999;341:1565-1571.

27. Rajkumar SV, Blood E, Vesole D, Fonseca R, Greipp PR; Eastern Cooperative Oncology Group. Phase III clinical trial of thalidomide plus dexamethasone compared with dexamethasone alone in newly diagnosed multiple myeloma: a clinical trial coordinated by the Eastern Cooperative Oncology Group. J Clin Oncol 2006;24:431-436.

28. Richardson PG, Barlogie B, Berenson J, et al. A phase 2 study of bortezomib in relapsed, refractory myeloma. $\mathrm{N}$ Engl J Med 2003;348:2609-2617.

29. Richardson PG, Sonneveld P, Schuster MW, et al. Bortezomib or high-dose dexamethasone for relapsed multiple myeloma. N Engl J Med 2005;352:2487-2498.

30. Orlowski RZ, Nagler A, Sonneveld P, et al. Randomized phase III study of pegylated liposomal doxorubicin plus bortezomib compared with bortezomib alone in relapsed or refractory multiple myeloma: combination therapy improves time to progression. J Clin Oncol 2007;25:38923901.

31. San Miguel JF, Schlag R, Khuageva NK, et al. Bortezomib plus melphalan and prednisone for initial treatment of multiple myeloma. N Engl J Med 2008;359:906-917.

32. Sonneveld P, Goldschmidt H, Rosinol L, et al. Bortezomib-based versus nonbortezomib-based induction treatment before autologous stem-cell transplantation in patients with previously untreated multiple myeloma: a meta-analysis of phase III randomized, controlled trials. J Clin Oncol 2013;31:3279-3287.

33. Dimopoulos M, Spencer A, Attal M, et al. Lenalidomide plus dexamethasone for relapsed or refractory multiple myeloma. N Engl J Med 2007;357:2123-2132.

34. Weber DM, Chen C, Niesvizky R, et al. Lenalidomide plus dexamethasone for relapsed multiple myeloma in North America. N Engl J Med 2007;357:2133-2142.

35. Benboubker L, Dimopoulos MA, Dispenzieri A, et al. Lenalidomide and dexamethasone in transplant-ineligible patients with myeloma. N Engl J Med 2014;371:906917.

36. McCarthy PL, Owzar K, Hofmeister CC, et al. Lenalidomide after stem-cell transplantation for multiple myeloma. N Engl J Med 2012;366:1770-1781.

37. Kumar SK, Lee JH, Lahuerta JJ, et al. Risk of progression and survival in multiple myeloma relapsing after therapy with IMiDs and bortezomib: a multicenter international myeloma working group study. Leukemia 2012;26:149-157.

38. San Miguel J, Weisel K, Moreau P, et al. Pomalidomide plus low-dose dexamethasone versus high-dose dexamethasone alone for patients with relapsed and refractory multiple myeloma (MM-о03): a randomised, open-label, phase 3 trial. Lancet Oncol 2013;14:1055-1066. 
39. Kortuem KM, Stewart AK. Carfilzomib. Blood 2013;121:893897.

40. Stewart AK, Rajkumar SV, Dimopoulos MA, et al. Carfilzomib, lenalidomide, and dexamethasone for relapsed multiple myeloma. N Engl J Med 2015;372:142-152.

41. Dimopoulos MA, Moreau P, Palumbo A, et al. Carfilzomib and dexamethasone versus bortezomib and dexamethasone for patients with relapsed or refractory multiple myeloma (ENDEAVOR): a randomised, phase 3, open-label, multicentre study. Lancet Oncol 2016;17:27-38.

42. Moreau P, Masszi T, Grzasko N, et al. Ixazomib, an investigational oral proteasome inhibitor (PI), in combination with lenalidomide and dexamethasone (IRd), significantly extends progression-free survival (PFS) for patients (Pts) with relapsed and/or refractory multiple myeloma (RRMM): the phase 3 tourmaline-MMi study. Blood 2015;126:727.

43. Moreau P. Oral therapy for multiple myeloma: ixazomib arriving soon. Blood 2014;124:986-987.

44. San-Miguel JF, Hungria VT, Yoon SS, et al. Panobinostat plus bortezomib and dexamethasone versus placebo plus bortezomib and dexamethasone in patients with relapsed or relapsed and refractory multiple myeloma: a multicentre, randomised, double-blind phase 3 trial. Lancet Oncol 2014;15:1195-1206.

45. Lonial S, Dimopoulos M, Palumbo A, et al. Elotuzumab therapy for relapsed or refractory multiple myeloma. N Engl J Med 2015;373:621-631.

46. Lokhorst HM, Plesner T, Laubach JP, et al. Targeting $\mathrm{CD}_{3} 8$ with daratumumab monotherapy in multiple myeloma. N Engl J Med 2015;373:1207-1219.

47. Mateos MV, Hernandez MT, Giraldo P, et al. Lenalidomide plus dexamethasone for high-risk smoldering multiple myeloma. N Engl J Med 2013;369:438-447.

48. Dispenzieri A, Stewart AK, Chanan-Khan A, et al. Smoldering multiple myeloma requiring treatment: time for a new definition? Blood 2013;122:4172-4181.

49. International Myeloma Working Group. Criteria for the classification of monoclonal gammopathies, multiple myeloma and related disorders: a report of the International Myeloma Working Group. Br J Haematol 2003;121:749-757.

50. Ghobrial IM, Landgren O. How I treat smoldering multiple myeloma. Blood 2014;124:3380-3388.

51. Kyle RA, Remstein ED, Therneau TM, et al. Clinical course and prognosis of smoldering (asymptomatic) mul- tiple myeloma. N Engl J Med 2007;356:2582-259o.

52. Landgren O. Multiple myeloma precursor disease: current clinical dilemma and future opportunities. Semin Hematol 2011;48:1-3.

53. Rajkumar SV, Larson D, Kyle RA. Diagnosis of smoldering multiple myeloma. N Engl J Med 2011;365:474-475.

54. Kastritis E, Terpos E, Moulopoulos L, et al. Extensive bone marrow infiltration and abnormal free light chain ratio identifies patients with asymptomatic myeloma at high risk for progression to symptomatic disease. Leukemia 2013;27:947-953.

55. Larsen JT, Kumar SK, Dispenzieri A, Kyle RA, Katzmann JA, Rajkumar SV. Serum free light chain ratio as a biomarker for high-risk smoldering multiple myeloma. Leukemia 2013;27:941-946.

56. Hillengass J, Fechtner K, Weber MA, et al. Prognostic significance of focal lesions in whole-body magnetic resonance imaging in patients with asymptomatic multiple myeloma. J Clin Oncol 2010;28:1606-1610.

57. Weber DM, Dimopoulos MA, Moulopoulos LA, Delasalle KB, Smith T, Alexanian R. Prognostic features of asymptomatic multiple myeloma. Br J Haematol 1997;97:810814.

58. Regelink JC, Minnema MC, Terpos E, et al. Comparison of modern and conventional imaging techniques in establishing multiple myeloma-related bone disease: a systematic review. Br J Haematol 2013;162:50-61.

59. Hutchison CA, Batuman V, Behrens J, et al. The pathogenesis and diagnosis of acute kidney injury in multiple myeloma. Nat Rev Nephrol 2012;8:43-51.

6o. Avet-Loiseau H, Durie BG, Cavo M, et al. Combining fluorescent in situ hybridization data with ISS staging improves risk assessment in myeloma: an International Myeloma Working Group collaborative project. Leukemia 2013;27:711-717.

61. Palumbo A, Avet-Loiseau H, Oliva S, et al. Revised international staging system for multiple myeloma: a report from International Myeloma Working Group. J Clin Oncol 2015;33:2863-2869.

62. Kim C, Lee HS, Min CK, et al. The prognostic impact of inflammatory factors in patients with multiple myeloma treated with thalidomide in Korea. Korean J Intern Med 2015;30:675-683.

63. Mikhael JR, Dingli D, Roy V, et al. Management of newly diagnosed symptomatic multiple myeloma: updated Mayo Stratification of Myeloma and Risk-Adapted Thera- 
py (mSMART) consensus guidelines 2013. Mayo Clin Proc 2013;88:360-376.

64. Chng WJ, Dispenzieri A, Chim CS, et al. IMWG consensus on risk stratification in multiple myeloma. Leukemia 2014;28:269-277.

65. Kuiper R, van Duin M, van Vliet MH, et al. Prediction of high- and low-risk multiple myeloma based on gene expression and the International Staging System. Blood 2015;126:1996-2004.

66. Palumbo A, Bringhen S, Mateos MV, et al. Geriatric assessment predicts survival and toxicities in elderly myeloma patients: an International Myeloma Working Group report. Blood 2015;125:2068-2074.

67. Lee H, Lee M. A case of alphaz plasmacytoma. New Med J 1959;2:1113-1117.

68. Kang DY, Lee DI, Kim KH, et al. Statistical studies on multiple myeloma in Korea: preliminary report. Korean J Hematol 1972;7:31-40.

69. Kim HJ, Heo DS, Bang YJ, et al. Multiple myeloma in Korea: clinical analysis and treatment results in 61 cases. Korean J Med 1985;28:770-779.

70. Jung JW, Kim JH, Kim SY, Yoon HJ, Cho KS. A clinical study on multiple myelom. J Korean Cancer Assoc 1995;27:869-879.

71. Kim TY, Heo DS, Bang YJ, et al. Combination chemotherapy with vincristine, melphalan, and prednisolone for multiple myeloma. Korean J Med 1993;45:1-12.

72. Kim HJ, Seo CI, Park KC, et al. Combination chemotherapy for the treatment of multiple myeloma. J Korean Cancer Assoc 1992;24:577-585.

73. Lee JT, Kim IH, Ahn JS, et al. Phase 2 trial of VAD (vincristine, doxorubicin, and dexamethasone) in refractory multiple myeloma. Korean J Hematol 1996;31:145-153.

74. Lee JH, Bang SM, Lee S, et al. High dose chemotherapy with autologous stem cell transplantation in multiple myeloma. Korean J Hematol 1999;34:306-316.

75. Bang SM, Cho EK, Suh C, et al. High dose therapy followed by autologous peripheral blood stem cell transplantation as a first line treatment for multiple myeloma: a Korean Multicenter Study. J Korean Med Sci 2003;18:673-678.

76. Bang SM, Lee JH, Yoon SS, et al. Preliminary report of risk-based approach in Korean patients with newly diagnosed multiple myeloma. Blood 2004;104:4911.

77. Lee KW, Yun T, Song EK, et al. A pilot study of bortezomib in Korean patients with relapsed or refractory myeloma. J
Korean Med Sci 2005;20:598-562.

78. Kim DY, Im SA, Seong CM, et al. Salvage therapy with thalidomide in patients with multiple myeloma. Korean J Hematol 2002;37:259-264.

79. Oh HS, Choi JH, Park CK, et al. Comparison of microvessel density before and after peripheral blood stem cell transplantation in multiple myeloma patients and its clinical implications: multicenter trial. Int J Hematol 2002;76:465-470.

8o. Bang SM, Lee JH, Yoon SS, et al. A multicenter retrospective analysis of adverse events in Korean patients using bortezomib for multiple myeloma. Int J Hematol 2006;83:309-313.

81. Kim HJ, Yoon SS, Lee DS, et al. Sequential vincristine, adriamycin, dexamethasone (VAD) followed by bortezomib, thalidomide, dexamethasone (VTD) as induction, followed by high-dose therapy with autologous stem cell transplant and consolidation therapy with bortezomib for newly diagnosed multiple myeloma: results of a phase II trial. Ann Hematol 2012;91:249-256.

82. Yang DH, Kim YK, Sohn SK, et al. Induction treatment with cyclophosphamide, thalidomide, and dexamethasone in newly diagnosed multiple myeloma: a phase II study. Clin Lymphoma Myeloma Leuk 2010;10:62-67.

83. Kim JS, Suh C, Cheong JW, et al. Sequential high-dose dexamethasone and response adapted PAD (bortezomib, adriamycin, dexamethasone) or VAD (vincristine, adriamycin, dexamethasone) induction chemotherapy followed by high-dose chemotherapy with autologous stem cell transplantation for newly diagnosed multiple myeloma: open-labelled, multicenter phase 2 study (KMM-94 study): interim analysis. Blood 2011;118:2044.

84. Eom HS, Kim YK, Chung JS, et al. Bortezomib, thalidomide, dexamethasone induction therapy followed by melphalan, prednisolone, thalidomide consolidation therapy as a first line of treatment for patients with multiple myeloma who are non-transplant candidates: results of the Korean Multiple Myeloma Working Party (KMMWP). Ann Hematol 2010;89:489-497.

85. Lee HS, Kim YS, Kim K, et al. Early response to bortezomib combined chemotherapy can help predict survival in patients with multiple myeloma who are ineligible for stem cell transplantation. J Korean Med Sci 2013;28:80-86.

86. Lee SS, Suh C, Kim BS, et al. Bortezomib, doxorubicin, and dexamethasone combination therapy followed by thalidomide and dexamethasone consolidation as a 
salvage treatment for relapsed or refractory multiple myeloma: analysis of efficacy and safety. Ann Hematol 2010;89:905-912.

87. Kim YK, Sohn SK, Lee JH, et al. Clinical efficacy of a bortezomib, cyclophosphamide, thalidomide, and dexamethasone (Vel-CTD) regimen in patients with relapsed or refractory multiple myeloma: a phase II study. Ann Hematol 2010;89:475-482.

88. Kim K, Kim SJ, Voelter V, et al. Lenalidomide with dexamethasone treatment for relapsed/refractory myeloma patients in Korea-experience from 110 patients. Ann Hematol 2014;93:113-121.

89. Kim JS, Kim K, Cheong JW, et al. Complete remission status before autologous stem cell transplantation is an important prognostic factor in patients with multiple myeloma undergoing upfront single autologous transplantation. Biol Blood Marrow Transplant 2009;15:463-470.

90. Min CK, Kim H, Kim K, et al. A multicenter comparison of autologous stem cell transplantation followed by reduced-intensity allogenic stem cell transplantation with tandem autologous stem cell transplantation in multiple myeloma. Blood 2007;110:940.

91. Kim SJ, Kim K, Kim BS, et al. Clinical features and survival outcomes in patients with multiple myeloma: analysis of web-based data from the Korean Myeloma Registry. Acta Haematol 2009;122:200-210.

92. Oh S, Koo DH, Kwon MJ, et al. Chromosome 13 deletion and hypodiploidy on conventional cytogenetics are robust prognostic factors in Korean multiple myeloma patients: web-based multicenter registry study. Ann Hematol 2014;93:1353-1361.

93. Kim HJ, Choi DR, Yun GW, et al. Myelomatous pleural effusion of multiple myeloma: characteristics and outcome. Blood 2009;114:3874.

94. Kim MK, Suh C, Lee DH, et al. Immunoglobulin D multiple myeloma: response to therapy, survival, and prognostic factors in 75 patients. Ann Oncol 2011;22:411-416.

95. Bang SM, Seo JW, Park KU, et al. Molecular cytogenetic analysis of Korean patients with Waldenstrom macroglobulinemia. Cancer Genet Cytogenet 2010;197:117-121.

96. Jun HJ, Kim K, Kim SJ, et al. Clinical features and treatment outcome of primary systemic light-chain amyloidosis in Korea: results of multicenter analysis. Am J Hematol 2013;88:52-55.

97. Kim HJ, Shin H, Park EK, et al. Osteonecrosis of the jaw in multiple myeloma patients: incidence and characteris- tics in Korean patients. Blood 2009;114:4956.

98. Kim SJ, Kim K, Kim BS, et al. Bortezomib and the increased incidence of herpes zoster in patients with multiple myeloma. Clin Lymphoma Myeloma 2008;8:237-240.

99. Kim SJ, Kim K, Do YR, Bae SH, Yang DH, Lee JJ. Low-dose acyclovir is effective for prevention of herpes zoster in myeloma patients treated with bortezomib: a report from the Korean Multiple Myeloma Working Party (KMMWP) Retrospective Study. Jpn J Clin Oncol 2011;41:353-357.

100. Koh Y, Bang SM, Lee JH, et al. Low incidence of clinically apparent thromboembolism in Korean patients with multiple myeloma treated with thalidomide. Ann Hematol 2010;89:201-206.

101. Bang SM, Kim YR, Cho HI, et al. Identification of 139 deletion, trisomy 1q, and $\mathrm{IgH}$ rearrangement as the most frequent chromosomal changes found in Korean patients with multiple myeloma. Cancer Genet Cytogenet 2006;168:124-132.

102. Kang SH, Kim TY, Kim HY, et al. Association of NQO1 polymorphism with multiple myeloma risk in Koreans. Korean J Lab Med 2006;26:71-76.

103. Kang SH, Kim TY, Kim HY, et al. Protective role of CY$\mathrm{P}_{1} \mathrm{~A}_{1}{ }_{2} \mathrm{~A}$ in the development of multiple myeloma. Acta Haematol 2008;119:60-64.

104. Kim TY, Park J, Oh B, et al. Natural polyphenols antagonize the antimyeloma activity of proteasome inhibitor bortezomib by direct chemical interaction. Br J Haematol 2009;146:270-281.

105. Kim SY, Min HJ, Park HK, et al. Increased copy number of the interleukin- 6 receptor gene is associated with adverse survival in multiple myeloma patients treated with autologous stem cell transplantation. Biol Blood Marrow Transplant 2011;17:810-820.

106. Park G, Kang SH, Lee JH, et al. Concurrent p16 methylation pattern as an adverse prognostic factor in multiple myeloma: a methylation-specific polymerase chain reaction study using two different primer sets. Ann Hematol 2011;90:73-79.

107. Lee JJ, Choi BH, Kang HK, et al. Induction of multiple myeloma-specific cytotoxic T lymphocyte stimulation by dendritic cell pulsing with purified and optimized myeloma cell lysates. Leuk Lymphoma 2007;48:2022-2031.

108. Kim SK, Nguyen Pham TN, Nguyen Hoang TM, et al. Induction of myeloma-specific cytotoxic $\mathrm{T}$ lymphocytes ex vivo by $\mathrm{CD}_{40} \mathrm{O}-$ activated B cells loaded with myeloma tumor antigens. Ann Hematol 2009;88:1113-1123. 
109. Yang DH, Park JS, Jin CJ, et al. The dysfunction and abnormal signaling pathway of dendritic cells loaded by tumor antigen can be overcome by neutralizing VEGF in multiple myeloma. Leuk Res 2009;33:665-670.

110. Yang DH, Kim MH, Hong CY, et al. Alpha-type 1-polarized dendritic cells loaded with apoptotic allogeneic myeloma cell line induce strong CTL responses against autologous myeloma cells. Ann Hematol 2010;89:795-801.

111. Avet-Loiseau H, Durie BG, Haessler J, et al. Impact of FISH and cytogenetics on overall and event free survival in myeloma: an IMWG analysis of 9,897 patients. Blood 2009;114:743.

112. Palumbo A, Sezer O, Kyle R, et al. International Myeloma Working Group guidelines for the management of multiple myeloma patients ineligible for standard high-dose chemotherapy with autologous stem cell transplantation. Leukemia 2009;23:1716-1730.

113. Richardson PG, Delforge M, Beksac M, et al. Manage- ment of treatment-emergent peripheral neuropathy in multiple myeloma. Leukemia 2012;26:595-608.

114. Kim K, Lee JH, Kim JS, et al. Clinical profiles of multiple myeloma in Asia-An Asian Myeloma Network study. Am J Hematol 2014;89:751-756.

115. Tan D, Chng WJ, Chou T, et al. Management of multiple myeloma in Asia: resource-stratified guidelines. Lancet Oncol 2013;14:e571-e581.

116. Tan D, Kim K, Kim JS, et al. The impact of upfront versus sequential use of bortezomib among patients with newly diagnosed multiple myeloma (MM): a joint analysis of the Singapore MM Study Group and the Korean MM Working Party for the Asian Myeloma Network. Leuk Res 2013;37:1070-1076.

117. Baz R, Lin HY, Yoon SS, et al. Response adapted lenalidomide based therapy for newly diagnosed (ND) standard risk older adults with multiple myeloma (MM): an international collaboration. Blood 2013;122:3201. 